\title{
Self-assembled flower-like nanostructures of InN and GaN grown by plasma-assisted molecular beam epitaxy
}

\author{
MAHESH KUMAR ${ }^{1,2}$, T N BHAT ${ }^{1}$, M K RAJPALKE ${ }^{1}$, B ROUL ${ }^{1,2}$, P MISRA $^{3}$, \\ L M KUKREJA ${ }^{3}$, NEERAJ SINHA ${ }^{4}$, A T KALGHATGI ${ }^{2}$ and $S$ B KRUPANIDHI,* \\ ${ }^{1}$ Materials Research Centre, Indian Institute of Science, Bangalore 560 012, India \\ ${ }^{2}$ Central Research Laboratory, Bharat Electronics, Bangalore 560 013, India \\ ${ }^{3}$ Laser Materials Processing Division, Raja Ramanna Centre for Advanced Technology, Indore 452 013, India \\ ${ }^{4}$ Office of Principal Scientific Advisor, Government of India, New Delhi 110 011, India
}

MS received 18 March 2010

\begin{abstract}
Nanosized hexagonal InN flower-like structures were fabricated by droplet epitaxy on GaN/ $\mathrm{Si}(111)$ and $\mathrm{GaN}$ flower-like nanostructure fabricated directly on $\mathrm{Si}(111)$ substrate using radio frequency plasma-assisted molecular beam epitaxy. Powder X-ray diffraction (XRD) and scanning electron microscopy (SEM) were used to study the crystallinity and morphology of the nanostructures. Moreover, $X$-ray photoelectron spectroscopy (XPS) and photoluminescence (PL) were used to investigate the chemical compositions and optical properties of nano-flowers, respectively. Activation energy of free exciton transitions in GaN nano-flowers was derived to be $\sim 28.5 \mathrm{meV}$ from the temperature dependent PL studies. The formation process of nanoflowers is investigated and a qualitative mechanism is proposed.
\end{abstract}

Keywords. Nitrides; nano-flowers; photoluminescence; RF-MBE.

\section{Introduction}

Zero-dimension and one-dimension structures of indium nitride and gallium nitride are known to have great prospects in fundamental physical science and novel technological applications (Morkoc and Mohammad 1995; Ponce and Bour 1997). Because of the large bandgap of $\mathrm{GaN}$ and small bandgap of InN and size confinements in such nanostructures, the fabrication of infrared, visible and ultra-violet light emitting devices (LED) with relatively low power consumption is potentially feasible (Takeuchi et al 1998; Matsuoka et al 2002; Wu et al 2002; Chen et al 2006). These studies mainly focused on zero-dimension quantum dot, one-dimensional nanowires or nanorods and two-dimensional quantum well structures. The investigation of combined zero and onedimensional nanometre-scale InN and GaN flowers could enable unique opportunities in understanding fundamental concepts underlying the observed optical, electronic and mechanical properties. In growing pure $\mathrm{InN}$ and $\mathrm{GaN}$, the main difficulty lies with their thermal decomposition, which becomes excessive above $500^{\circ} \mathrm{C}$ for $\mathrm{InN}$ and $800^{\circ} \mathrm{C}$ for $\mathrm{GaN}$, and its impurity incorporation problem, especially by oxygen (Wang and Yoshikawa 2004). Plasma-assisted molecular beam epitaxy (MBE) is a suitable method for producing InN and $\mathrm{GaN}$, because low

*Author for correspondence (sbk@mrc.iisc.ernet.in) growth temperatures are possible in combination with an ultra high vacuum (UHV) growth environment, thus reducing the impurity incorporation (Lu et al 2001; Nanishi et al 2003). To fabricate III-nitride nanostructures by Stranski-Krastanow (SK) growth mode (Adelmann et al 2002), recently droplet epitaxy (DE) technique has been utilized (Maruyama et al 2007). In DE technique, to convert the droplets into semiconductor nanostructures, group III droplets are exposed to a subsequent group V molecular beam in DE-MBE approach. During this process, liquid metal droplet can be modified into various shapes of nanostructures (Lee et al 2009). The vapour phase growth mechanisms of nanowires include vapoursolid (VS) and vapour-liquid-solid (VLS). Contrary to the restricted growth of nanowires from the metal catalysts by VLS mechanism, the VS mechanism can give more freedom in the formation of nano-flowers.

In this study, InN nano-flowers structures on GaN/ $\mathrm{Si}(111)$ substrates were grown by droplet epitaxy technique and GaN nano-flower structures on Si (111) substrate by VS mechanism using an RF plasma-assisted MBE.

\section{Experimental}

The growth system employed in the present study was RF-MBE system (OMICRON) equipped with a radio frequency $(\mathrm{RF})$ plasma source. The base pressure in the system was below $1 \times 10^{-10}$ mbar. The undoped silicon 
(111) wafers, as substrate, were loaded into the MBE system and out-gassed at $900^{\circ} \mathrm{C}$ for $1 \mathrm{~h}$ to remove the native oxide. Then, $250 \mathrm{~nm}$ of $\mathrm{GaN}$ films were grown directly on the substrate. After GaN growth, nitrogen plasma was switched on for $10 \mathrm{~min}$ at $540^{\circ} \mathrm{C}$ substrate temperature, forming a nitrogen rich surface. The plasma power and nitrogen flow rate were kept at $350 \mathrm{~W}$ and $0.7 \mathrm{sccm}$, respectively. The sample was then exposed to an indium (In) molecular beam at $160^{\circ} \mathrm{C}$ just above the melting point of In for $180 \mathrm{~s}$. In cell, temperature was kept at $830^{\circ} \mathrm{C}$ and corresponding beam equivalent pressure (BEP) was $6.7 \times 10^{-7}$ mbar. Next, the In droplets were exposed for $30 \mathrm{~min}$ with nitrogen plasma for nitridation of the In droplets. The nitrogen flow rate and plasma power were $0.7 \mathrm{sccm}$ and $350 \mathrm{~W}$, respectively. In addition, a post-growth annealing at $400^{\circ} \mathrm{C}$ was carried out for $30 \mathrm{~min}$. This sample was labeled sample A. In sample $\mathrm{B}$, after thermal cleaning of $\mathrm{Si}(111)$, the sample was exposed to gallium $(\mathrm{Ga})$ molecular beam at $800^{\circ} \mathrm{C}$ for $60 \mathrm{~s}$. Afterwards, nitrogen plasma and Ga flux were

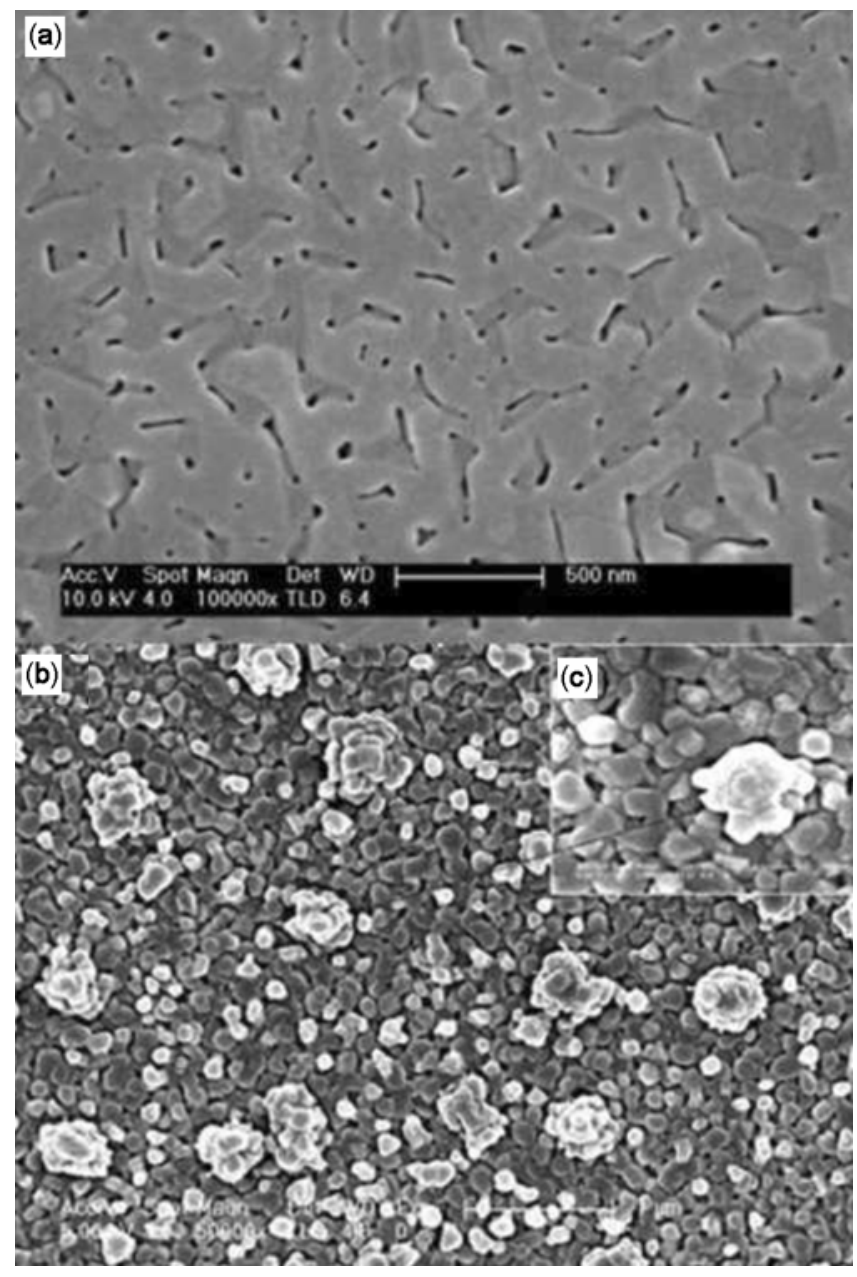

Figure 1. SEM images of (a) GaN surface on Si(111) substrate, (b) and (c) InN flower-like nanostructures grown on GaN/ $\mathrm{Si}(111)$ at different magnifications $(\times 50000$ and $\times 200000$, respectively). switched simultaneously for $60 \mathrm{~min}$ at $800^{\circ} \mathrm{C}$ substrate temperature, which led to the formation of GaN flowerlike structures. The plasma power and nitrogen flow rate were kept at $350 \mathrm{~W}$ and $0.7 \mathrm{sccm}$, respectively and $\mathrm{Ga}$ cell temperature was kept at $950^{\circ} \mathrm{C}$ and corresponding $\mathrm{BEP}$ was $5.6 \times 10^{-7}$ mbar.

The structural characterization, surface morphologies and chemical composition studies were carried out by XRD, SEM and XPS. Besides, the flower-like nanostructures were characterized by temperature dependent PL spectroscopy using the $325 \mathrm{~nm}$ line of a $\mathrm{He}-\mathrm{Cd}$ laser as an excitation source.

\section{Results and discussion}

Figure 1 shows the surface morphology of the GaN and as-grown InN nanostructures on the $c$-plane $\mathrm{GaN} / \mathrm{Si}(111)$ substrates. From the SEM observations, it is evident that the deposited InN nanostructures have flower-like morphology, in between flowers and each flower-like nanostructure contains few leaf-like InN nanoflakes. GaN flowerlike nanostructures are shown in figure 2 at different magnifications.

Figure 3 shows $2 \theta / \omega$ XRD pattern of the InN flowerlike nanostructures grown on $c$-plane $\mathrm{GaN}$ on $\mathrm{Si}(111)$ and GaN flower-like nanostructures grown on $\mathrm{Si}(111)$ substrate. In sample $\mathrm{A}$, the diffraction peaks appearing at $31.226^{\circ}$ and $34.662^{\circ}$ are assigned to the $\operatorname{InN}(0002)$ and $\mathrm{GaN}$ (0002) diffractions of the wurtzite-type structures, in which the peak appearing at $28.482^{\circ}$ is due to the $\mathrm{Si}(111)$ diffraction. In sample $B$, the diffraction peak appearing at $34.662^{\circ}$ is assigned to the (0002) diffractions of the wurtzite-type structure of GaN. In addition, the

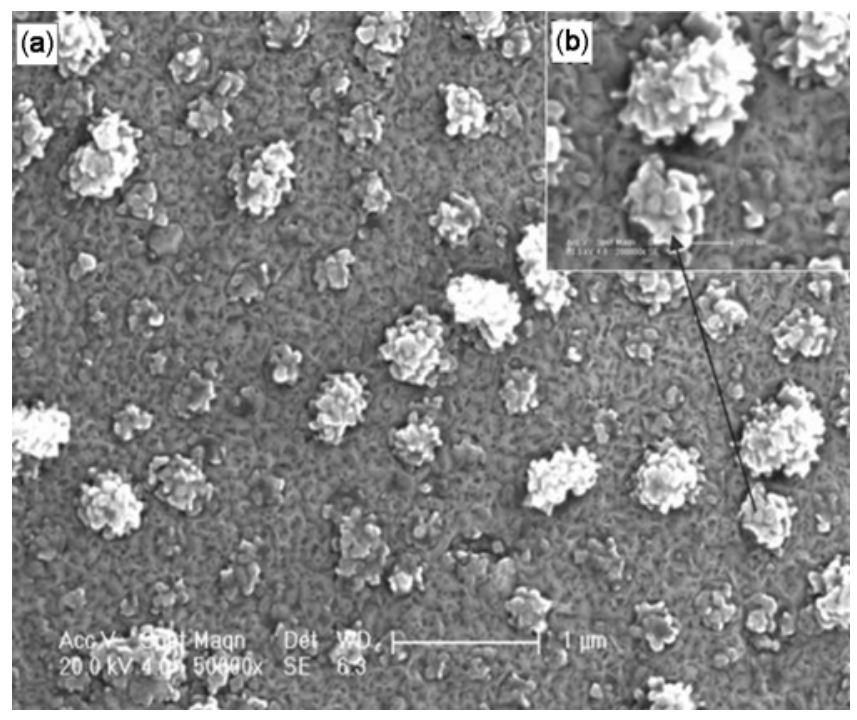

Figure 2. SEM images of flower-like $\mathrm{GaN}$ nanostructures grown on $\mathrm{Si}(111)$ : (a) magnification, $\times 50,000$ and (b) magnification $\times 2,00,000$. 
XRD of the nanostructures also shows features typical of nanodimensional particles. The $\mathrm{InN}(0002)$ and $\mathrm{GaN}$ (0002) planes of wurtzite structures are not well defined, appearing as a broad peak, indicating the small size of particles. According to the line width analysis of the (0002) diffraction peak based on the Scherrer formula, the InN and GaN flower-like structures are on the nanometer scale and an average crystallite size of about 11.21 and $10.87 \mathrm{~nm}$ was roughly estimated, respectively.

The XPS spectrum was acquired using $\mathrm{AlK}_{\alpha}$ radiation $(h v=1486.6 \mathrm{eV})$. Figures 4(a)-(b) show the In $3 d$ and $\mathrm{N}$ 1s XPS spectra of InN nano-flowers, respectively. All the binding energies were corrected with contamination $\mathrm{C} 1 s$ signal $(285.0 \mathrm{eV})$. The In $3 d$ core is spin-orbit split to the $3 d_{5 / 2}$ at $444.2 \mathrm{eV}$ and $3 d_{3 / 2}$ peak at $451.7 \mathrm{eV}$. The peak at around $396.5 \mathrm{eV}$ corresponds to N1 $s$ of InN nanostructures. These results are close to the reported values of InN films (Lu et al 1993; Parala et al 2001). Figures 4(c)-(d) exhibit the binding energy peaks of Ga3d at $20.2 \mathrm{eV}$ and of $\mathrm{N} 1 s$ at $397.2 \mathrm{eV}$ of GaN nanostructures, respectively and these values are very close to reported values of bulk GaN (Kowalski et al 2001, 2004).

The optical properties of the InN nano-flowers were characterized by low temperature PL measurements. The $10 \mathrm{~K}$ PL spectra of InN nanostructures, as shown in figure 5(a), was found to have peak energies centred at $0.720,0.782$ and $0.852 \mathrm{eV}$. Notably, the PL spectrum contained multiple peaks and slightly blue shifted compared to the bulk InN, which may be due to the presence

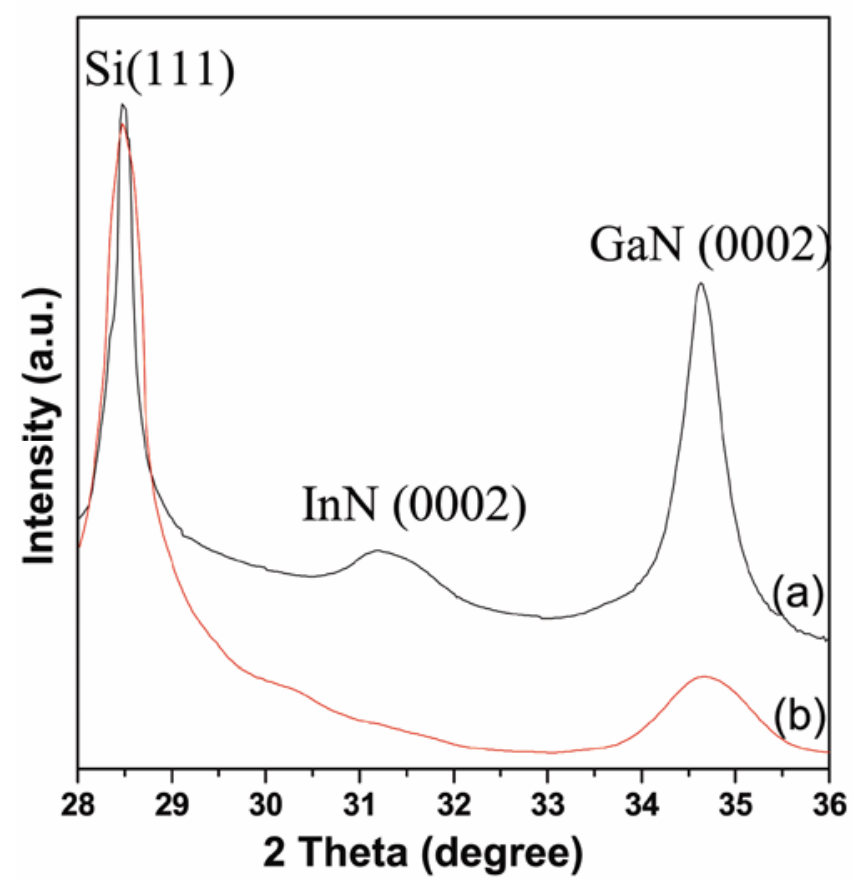

Figure 3. $2 \theta / \omega \mathrm{XRD}$ profile of the flower-like nanostructures of (a) InN grown on $c$-plane $\mathrm{GaN} / \mathrm{Si}(111)$ and (b) $\mathrm{GaN}$ grown on $\mathrm{Si}(111)$ substrate. of nano families with different particle sizes in the sample and the size dependent quantum confinement effect, respectively (Brusaferri et al 1996). Figure 5(b) shows temperature-dependent PL spectra of GaN nano-flowers, in the range of 5-295 K. The two emission lines, observed at low temperatures below $60 \mathrm{~K}$ were assigned to the donor bound exciton (DBE) emission which is merged with two-electron satellites (TES) of DBE and the free exciton (FX) emission. With increasing temperature, the intensity of the DBE peak decreased abruptly due to delocalization of the bound exciton, while that of the FX peak decreased gradually with temperature. This is the typical behaviour of free and bound exciton emissions commonly observed in epitaxial GaN (Chen et al 2006). In addition, the FX and DBE emission peaks of GaN nano-flowers were found to be slightly blue-shifted compared to bulk GaN, which was attributed to the quantum confinement effect (Ke et al 2006; Park et al 2008) in $\mathrm{GaN}$ nano flowers. The decrease of PL intensity with increasing temperature can be demonstrated by the thermal ionization of electron-hole bound states or localized states. The intensities of the FX transition peaks at different temperatures have been fitted with the generalized expression (Davies et al 1989)

$$
I(T)=I_{\mathrm{o}} /\left[1+C_{\mathrm{o}} \exp \left(-E_{\mathrm{a}} / k T\right)+C_{1} \exp \left(-E_{\mathrm{loc}} / k T\right)\right],
$$

where $I(T)$ is the PL intensity at temperature $T, C_{0}, C_{1}$ and $I_{\mathrm{o}}$ are constants, $k$ is Boltzmann's constant and $E_{\mathrm{a}}$ and $E_{\text {loc }}$ are the activation energy and localization energy in the high and low temperature regime, respectively.

Figure 6 shows Arrhenius plot for the peak PL intensities for the FX transition related PL emission as a function of temperature. We expect the PL intensity to be dominated by localized excitons at low temperature $(T<60 \mathrm{~K})$. At higher temperatures $(T>60 \mathrm{~K})$, the PL intensity is controlled by thermally activated centres. The fit gives, localization energy $\left(E_{\text {loc }}\right)$ to be $5.2 \mathrm{meV}$ and thermal activation energy $\left(E_{\mathrm{a}}\right)$ to be $28.5 \mathrm{meV}$. Thermal activation energy is slightly higher than that reported by Park et al (2008) who reported a value of $21 \mathrm{meV}$ and Chen et al (2006) and Chichibu et al (1996) who reported a value of $217 \mathrm{meV}$, perhaps due to the size dependent quantum confinement effect in the nano-flowers.

A schematic diagram of the growth mechanism of $\mathrm{InN}$ flower-like nanostructure is shown in figure 7. An In droplet on a planar GaN surface is shown schematically in figure 7(b). The net inward force per length applied to the drop at any point on the contact line is $\gamma_{1 \mathrm{~s}}-\gamma_{\mathrm{vs}}$, where $\gamma_{\mathrm{vs}}$ and $\gamma_{1 \mathrm{~s}}$ are the energies of the vacuum-solid and liquidsolid interfaces, respectively. (The force vector from the liquid surface tension, $\gamma_{\mathrm{vl}}$, sums to zero around the contact line, and so does not contribute to the net force on the droplet.) In a symmetric situation, this inward force vector has equal magnitude around the entire perimeter, so it integrates to zero total force on the drop. The nitridation at $160^{\circ} \mathrm{C}$ is converting In droplets into $\mathrm{InN}$ but 

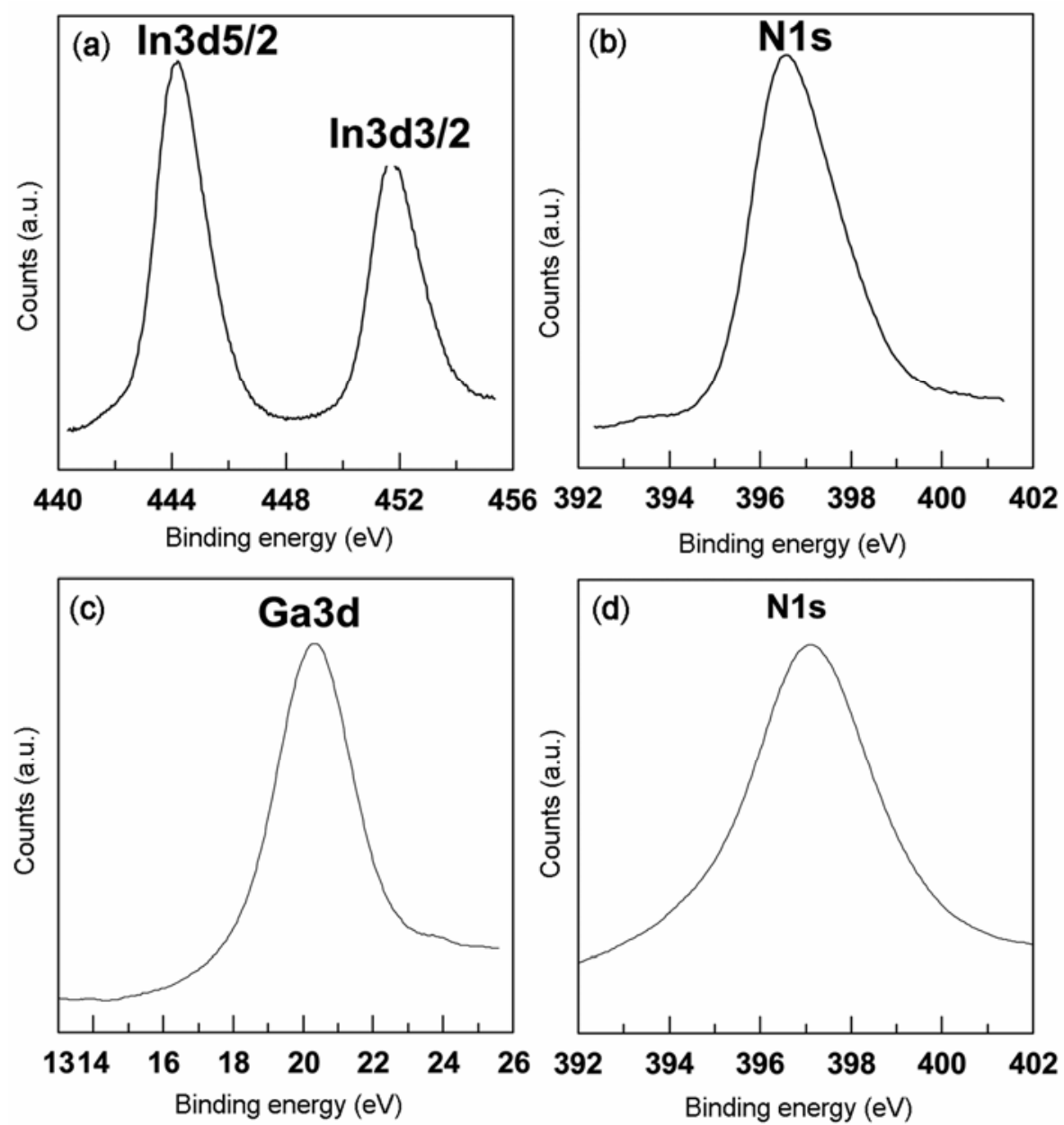

Figure 4. XPS spectra of (a) In $3 d$ and (b) N1s of InN nanostructures on GaN/Si (111), (c) Ga3 $d$ and (d) N1s of the GaN nanostructures.

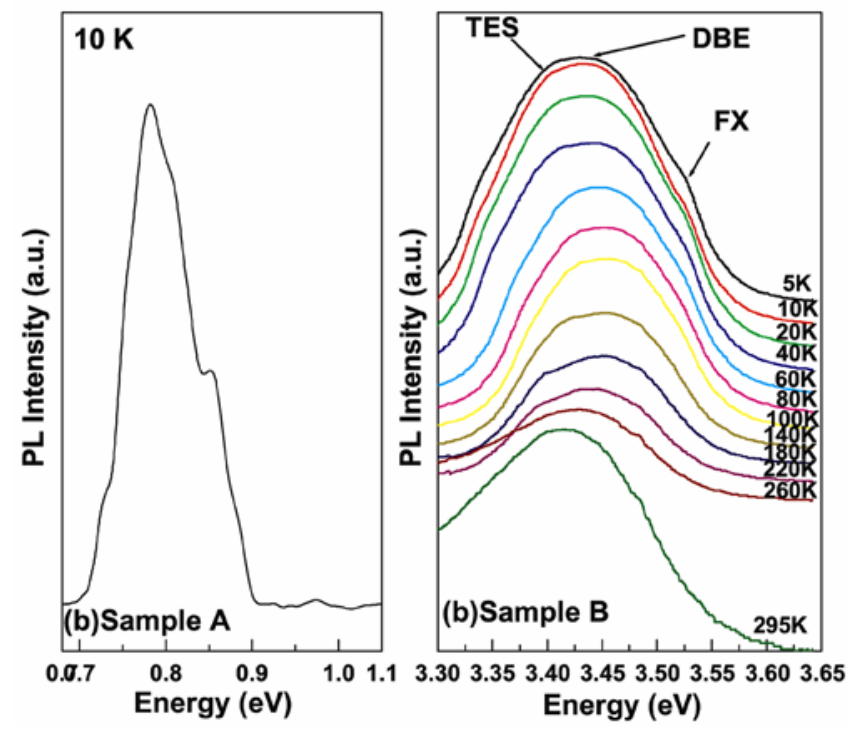

Figure 5. The PL spectra of the flower-like nanostructures of (a) InN grown on $c$-plane $\mathrm{GaN} / \mathrm{Si}$ (111) and (b) GaN grown on Si(111) substrate.

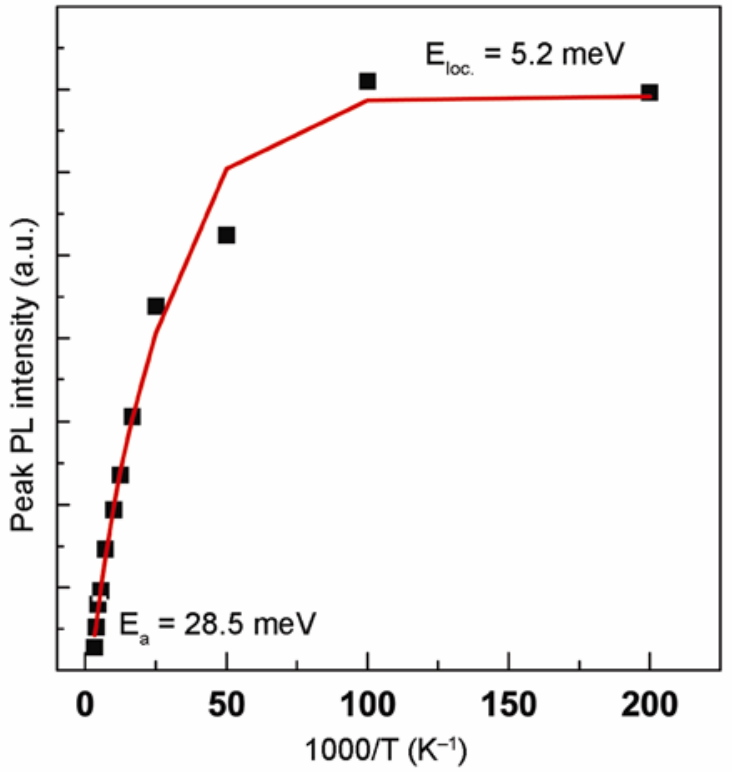

Figure 6. Peak PL intensities of FX transition as a function of temperature of the GaN nano-flowers. 


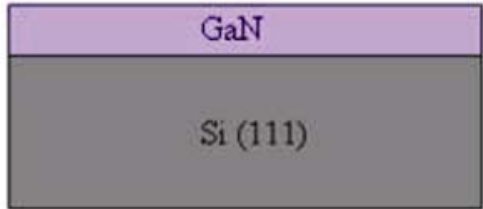

(a) GaN growth on $\mathrm{Si}(111)$

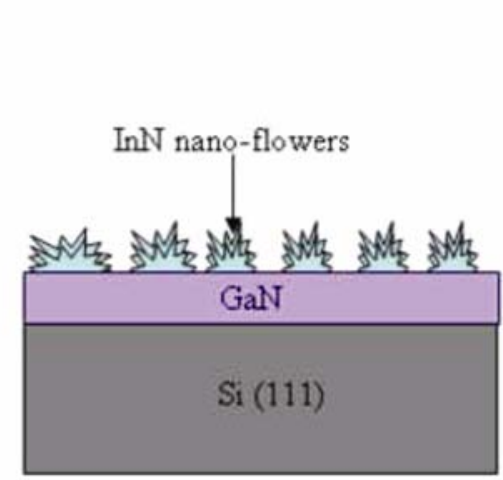

(d) Formation of nano-flowers

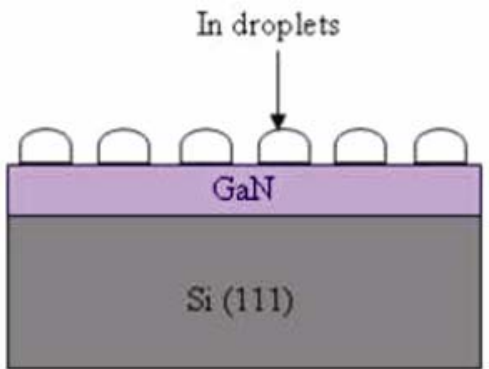

(b) Formation of In droplets

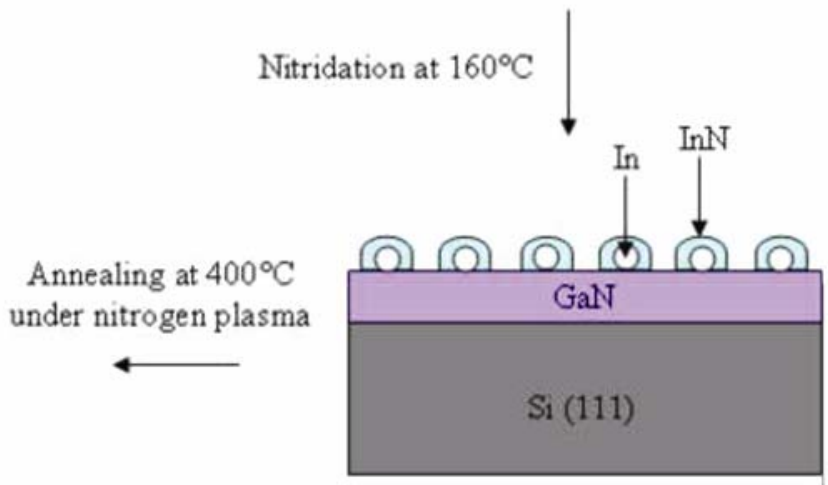

(c) Formation of InN

Figure 7. Schematic of growth mechanisms of InN nano-flowers on GaN/Si(111) substrate

due to surface diffusion limitation, In metal is not completely converting in InN as shown in figure 7(c).

Integrating the force vector around the perimeter, the total net force on the droplet is

$$
F_{\text {tot }}=\left(\gamma_{\text {ls(InN) }}-\gamma_{\text {ls(In) })}\right) d,
$$

where $d$ is the droplet diameter and $\gamma_{1 \mathrm{~s}(\mathrm{InN})}$ and $\gamma_{\mathrm{s}(\mathrm{In})}$ are the surface energies of liquid-solid interface of InN and In, respectively. If $\gamma_{1 \mathrm{~s}(\mathrm{In})}<\gamma_{\mathrm{s}(\mathrm{InN})}$, the droplet will remain stable but if $\gamma_{\mathrm{s}(\mathrm{In})}>\gamma_{\mathrm{vs}(\mathrm{InN})}$, the In will come out through InN cap layers in different shapes and minimizing the surface energy. The surface energies, $\gamma_{\mathrm{s}(\mathrm{InN})}$ and $\gamma_{\mathrm{s}(\mathrm{In})}$, depend on temperature and above a critical temperature, $\gamma_{1 \mathrm{~s}(\mathrm{In})}>\gamma_{\mathrm{vs}(\mathrm{InN})}$. When the substrate temperature increased from $160-400^{\circ} \mathrm{C}$ under nitrogen plasma, above critical temperature, liquid In came out through InN cap layers in nano-flower, minimizing the surface energy. During annealing at $400^{\circ} \mathrm{C}$ for $30 \mathrm{~min}$ under nitrogen plasma, In is completely converting in InN nano-flowers which is confirmed by our XRD analysis showing only hexagonal InN diffraction patterns. The GaN nano-flowers shown in figures 2(b)-(c) could be formed by the formation of small-sized GaN seed particles first and then growth of thin nanoflakes from the seeds. Many nanoflakes from the seeds can grow into a flower structure.

\section{Conclusions}

Self-assembled InN flower-like nanostructures on GaN/ $\mathrm{Si}$ (111) and GaN flower-like nanostructures on Si (111) have been grown successfully by RF MBE via noncatalytic route. The possible growth mechanism of InN and GaN nano-flowers are proposed. The nano-flowers were analysed by SEM, XRD and XPS. From the PL of $\mathrm{GaN}$ at low temperatures, the main emission lines were assigned as DBE and FE transitions which were blue shifted, due to the size confinement effect. The activation energy of FX emission lines of GaN nano-flowers was derived to be $28.5 \mathrm{meV}$. Thus the non-catalytic route provides a simple method to grow nano-flowers and gives freedom to fabricate a variety of nanostructures compared to the catalyst-confined vapour-solid-liquid (VLS) mechanism.

\section{References}

Adelmann C, Gogneau N, Sarigiannidou E, Rouviere J L and Daudin D 2002 Appl. Phys. Lett. 813064

Brusaferri L et al 1996 Appl. Phys. Lett. 693354

Chen H Y, Lin H W, Shen C H and Gwo S 2006 Appl. Phys. Lett. 89243105

Chichibu S, Azuhata T, Sota T and Nakamura S 1996 J. Appl. Phys. 792784 
Davies G 1989 Phys. Rep. 17683

Ke W C et al 2006 Nanotechnology 172609

Kowalski B J, Plucinski L, Kopalko K, Iwanowski R J, Orlowski B A, Johnson R L, Grzegory I and Porowski S 2001 Surf. Sci. 482-485 740

Kowalski B J, Iwanowski R J, Sadowski J, Kowalik I A, Kanski J, Grzegory I and Porowski S 2004 Surf. Sci. 548 220

Lee J H, Wang Z M, Kim N Y and Salamo G J 2009 Nanotechnology 20285602

Lu H, Schaff W J, Hwang J, Wu H, Koley G and Eastman L 2001 Appl. Phys. Lett. 791489

Lu Y, Ma L and Lin M C 1993 J. Vac. Sci. Technol. A11 2931

Maruyama T, Otsubo H, Kondo T, Yamamoto Y and Naritsuka S 2007 J. Cryst. Growth 301/302 486
Matsuoka T, Okamoto H, Nakao M, Harima H and Kurimoto E 2002 Appl. Phys. Lett. 811246

Morkoc H and Mohammad S N 1995 Science 26751

Nanishi Y, Saito Y and Yamaguchi T 2003 Jap. J. Appl. Phys. 422549

Parala H, Devi A, Hipler F, Maile E, Birkner A, Becker H W and Fischer R A 2001 J. Cryst. Growth 23168

Park Y S, Kang T W and Taylor R A 2008 Nanotechnology 19 475402

Ponce F A and Bour D P 1997 Nature 386351

Takeuchi T et al 1998 Appl. Phys. Lett. 731691

Wang X and Yoshikawa A 2004 Prog. Cryst. Growth Charact. Mater. 48/49 42

Wu J, Walukiewicz W, Yu K M, Ager III J W, Haller E E, Lu H, Schaff W J, Saito Y and Nanishi Y 2002 Appl. Phys. Lett. 803967 